\title{
Lesson of the month 1: Sodium valproate-induced encephalopathy
}

\author{
Author: Lara Camilleri
}

\begin{abstract}
A 69-year-old man developed reduced consciousness of sudden onset. Examination and parameters were normal, except for a Glasgow Coma Scale (GCS) score of six. Brain imaging and blood tests were also normal, except for high plasma ammonia. His past medical history included epilepsy, hypertension and colitis. He was taking multiple antiepileptic medications, including sodium valproate, with no recent dose alterations. Medical intervention led to the sodium valproate being stopped and naloxone being administered. The patient's level of responsiveness and ammonia levels gradually improved. The patient was also being treated with ciprofloxacin for a urinary tract infection and a newly developed syndrome of inappropriate antidiuretic hormone secretion treated with demeclocycline. There is an association between long-term sodium valproate use and low carnitine levels, especially in the setting of polypharmacy. This in turn precipitates hyperammonaemia and encephalopathy. This case highlights the importance of an adequate drug history and the awareness of serious but uncommon adverse effects.
\end{abstract}

KEYWORDS: Sodium valproate, hyperammonaemia, encephalopathy, naloxone

\section{Case summary}

A 69-year-old man was referred to the emergency department after he developed a reduced level of consciousness and paroxysmal breathing.

The patient was undergoing rehabilitation following a fractured femur. His significant past medical history included epilepsy secondary to superior sagittal sinus thrombosis, colitis with no current exacerbation, and hypertension. His drug history included: sodium valproate $500 \mathrm{mg}$ in the morning, $1 \mathrm{~g}$ in the evening; benzhexol $4 \mathrm{mg}$ daily; phenobarbital $1 \mathrm{~g}$ four times daily; carbamazepine $200 \mathrm{mg}$ three times daily; clopixol 200 mg monthly injections; calcium supplements twice daily; and enoxaparin $20 \mathrm{mg}$ daily subcutaneous injections. He had also been treated for the previous 6 days with ciprofloxacin for a pseudomonal urinary tract infection (UTI) and had started

Author: basic specialist trainee in general medicine, Mater Dei Hospital, Tal-Qroqq, Malta treatment with demeclocycline $300 \mathrm{mg} 3$ days previously because of newly developed syndrome of inappropriate antidiuretic hormone secretion (SIADH), which was still being investigated. There had been no recent dose adjustments to his antiepileptic treatment. He was a retired teacher living in a nursing home and was semi-dependent in personal activities of daily living. He did not have any cognitive impairment, drank alcohol socially and was an ex-smoker.

On examination, he was responsive to pain and noncommunicative. Parameters, including blood glucose, were stable. His chest was clear, heart sounds were normal and his abdomen was soft and nontender. His lower limbs had no signs of deep venous thrombosis. Neurologically, a left-sided mouth deviation was noted, power and sensation could not be assessed and muscle tone was flaccid throughout. His reflexes, including plantars, were normal, and his pupils were equal and reactive. Blood tests, including electrolytes, sodium valproate levels and arterial blood gasses, were normal. Computed tomography (CT) scan of the head was unremarkable. He was reviewed by a neurologist, who suspected metabolic encephalopathy; therefore, ammonia levels were determined and the patient started treatment with intravenous sodium valproate. However, his condition deteriorated and he developed stridor. Ammonia levels were elevated at $114 \mathrm{U} / \mathrm{L}$ (normal 7-55 U/L). The patient was not known to have liver disease, alcoholism, renal impairment or malignancy.

A literature review from the UpToDate application was done, and sodium valproate was listed as a possible cause of hyperammonaemia and encephalopathy, for which treatment with either naloxone or carnitine had been used in similar cases with good effect. At this point, naloxone $0.4 \mathrm{mg}$ was administered intravenously as a starting dose. The patient responded immediately; his Glasgow Coma Scale score improved and he regained his ability to communicate. He started treatment with a naloxone infusion of $2 \mathrm{mg}$ over 12 hours and was transferred to an acute medical ward on a monitored bed. He remained well off sodium valproate, which was later replaced with levetiracetam. His ammonia levels normalised gradually and his mental and functional abilities returned to normal.

\section{Discussion}

\section{Mechanism of action of sodium valproate}

The main component of sodium valproate is valproic acid (VPA), which is a branched short-chain fatty acid. Its main antiepileptic 
Table 1. Naloxone in valproate-induced encephalopathy

\begin{tabular}{|c|c|c|c|c|c|}
\hline Reference & Age (years) & Plasma valproate level & Additional treatment given & Naloxone effective? & Setting \\
\hline Thanacoody ${ }^{6}$ & 76 & $848 \mu \mathrm{mol} / \mathrm{L}$ & Ciprofloxacin for UTI & $\begin{array}{l}\text { Yes, within a few } \\
\text { minutes }\end{array}$ & Dehydration and UTI \\
\hline Alberto et $\mathrm{al}^{7}$ & 21 & $180 \mu \mathrm{g} / \mathrm{mL}$ & Multidose charcoal & Yes, within hours & Intentional overdose \\
\hline Eze et $a l^{8}$ & 69 & $107.2 \mu \mathrm{g} / \mathrm{mL}$ & $\begin{array}{l}\text { Lactulose enema and arbuterol } \\
\text { aerosol }\end{array}$ & Yes, within 1 day & Polypharmacy \\
\hline Bohmer et al ${ }^{9}$ & 68 & $254 \mu \mathrm{mol} / \mathrm{L}$ & L-carnitine effective & No, ineffective & $\begin{array}{l}\text { Postoperative } \\
\text { laparotomy for ileus }\end{array}$ \\
\hline
\end{tabular}

activities are: (i) inhibition of gamma-aminobutyric acid (GABA) transaminase enzyme, thereby increasing GABA levels in the brain; and (ii) blocking ion-gated voltage channels, thereby reducing the frequency of neuronal action potentials.

\section{Metabolism of sodium valproate}

VPA is metabolised in the liver via three main steps: (i) glucuronic acid conjugation within the liver; (ii) $\omega$-oxidation within the cytoplasm, resulting in the toxic metabolite 4 -en-VPA; and (iii) $\beta$-oxidation within the mitochondria, resulting in the non-toxic metabolite 2-en-VPA. Carnitine facilitates the transport of VPA into the mitochondria to promote $\beta$-oxidation. A by-product of $\beta$-oxidation is acetyl-CoA, which is used in the urea and Krebs cycles. $^{2}$

\section{Risk factors for valproate-induced encephalopathy}

The cause of valproate-induced encephalopathy secondary to hyperammonaemia can be multifactorial. ${ }^{3}$ There is a correlation between carnitine deficiency, urea cycle disorders and hyperammonaemia secondary to valproate. Carnitine deficiency can lead to a shift from $\beta$-oxidation towards $\omega$-oxidation, resulting in increased 4-en-VPA, which interferes with the urea cycle, thus increasing plasma ammonia levels. ${ }^{4}$

Carnitine is a nutrient found in meat and dairy products. Primary deficiency in carnitine and defects in the urea cycle can result from rare genetic conditions. Secondary carnitine deficiency can result from: (i) acquired medical conditions (eg chronic liver failure and renal failure); (ii) malabsorption or malnutrition (including in vegetarians, and those with coeliac and inflammatory bowel disease); (iii) a high catabolic state (eg trauma, malignancy or in acutely ill patients); and (iv) drugs, including chemotherapeutic agents, antiretrovirals, antiepileptics and antibiotics. ${ }^{4}$

VPA causes impaired tubular reabsorption of carnitine. Carnitine deficiency can result from both acute valproate toxicity and the following long-term use of sodium valproate despite normal plasma concentrations, especially when prescribed with other antiepileptic drugs or when concomitant antibiotics are given. ${ }^{4,5}$

In this case study, carnitine levels were not taken and ammonia levels normalised after naloxone and drug withdrawal. However, the patient was taking multiple antiepileptic drugs, had had a recent UTI treated with antibiotics and had recently started treatment with demeclocycline for SIADH; therefore, renal tubular absorption might have been affected further. He also had a history of colitis, which might result in malabsorption, although he had had no recent exacerbations.

\section{Naloxone in valproic acid-induced encephalopathy}

There are a few documented cases of the use of naloxone in the treatment of valproate-induced encephalopathy (Table 1). Its exact mode of action in this setting is still unclear but it is thought to result from its GABA antagonist effects. Naloxone also inhibits postsynaptic GABA transport because of VPA. ${ }^{10}$

\section{Conclusion}

Encephalopathy is an important differential diagnosis in patients presenting with an altered level of consciousness. In the absence of an obvious cause, it is crucial to review the patient's drug history and be familiar with the adverse effects of any medication. In this case, hyperammonaemia was an uncommon but important and possibly fatal adverse effect of sodium valproate. The medical literature indicates various treatment modalities that have been used in such cases, with L-carnitine being popular, given its role in valproate metabolism. In this case, naloxone and the withdrawal of sodium valproate were sufficient to normalise the patient's ammonia levels.

\section{Consent to publish}

Written consent was obtained from the patient to publish the clinical details and images in this article.

\section{References}

1 Ghodke-Puranik Y, Thorn CF, Lamba JK et al. Valproic acid pathway: pharmacokinetics and pharmacodynamics. Pharmacogenet Genomics 2013:23:236-41.

2 Vazquez M, Fagiolino P, Maldonado C et al. Hyperammonaemia associated with valproic acid concentrations. Biomed Res Int 2014;2014:217269.

3 Mittal V, Muralee S, Tampi RR. Valproic acid induced hyperammonaemia in the elderly: a review of the literature. Case Rep Med 2009;2009:802121.

4 Lheureux PE, Penaloza A, Zahir S, Gris M. Science review: Carnitine in the treatment of valproic acid induced toxicity - what is the evidence? Crit Care 2005;9:431-40.

5 Carr RB, Shrewsberry K. Hyperammonaemia due to valproic acid in the psychiatric setting. Am J Psychiatry 2007;164:1020-7. 
6 Thanacoody HK. Chronic valproic acid intoxication: reversal by naloxone. Emerg Med J 2007;24:677-8.

7 Alberto G, Erikson T, Popiel R, Narayanan M, Hryhorczuk D. Central nervous system manifestations of a valproic acid overdose responsive to naloxone. Ann Emerg Med 1989;18:889-91.

8 Eze E, Workeman M, Donley B. Hyperammonaemia and coma developed in woman treated with valproic acid for affective disorder. Psychiatr Serv 1998;49:1358-9.

9 Bohmer T, Bøem A, Hoymork C. Valproate-induced hyperammonemic encephalopathy rapidly induced by i.v. carnitine and glucose/thiamine. Scand J Gastroenterol 2010;45:762-3.
10 Dingledine R, Iversen LL, Breuker E. Naloxone as a GABA antagonist: evidence from iontopheric, receptor binding and convulsant studies. Eur ] Pharmacol 1978:47:19-27.

Address for correspondence: Dr Lara Camilleri, Mater Dei Hospital, Mazeltov, Dahlet Guzeppi Stagno, Marsaxlokk MXK1631, Malta.

Email: lara.camilleri@gov.mt

\title{
Lesson of the month 2: An unusual cause of depression in an older man: Cushing's syndrome resulting from metastatic small cell cancer of the prostate
}

\author{
Authors: Paul Kleinig ${ }^{\mathrm{A}, *}$ and Patrick Russell ${ }^{\mathrm{B}, *}$
}

This article describes the case of an older patient whose main presenting problem was depression. A constellation of clinical and biochemical features led to a search for medical causes of his low mood, which uncovered ectopic adrenocorticotropic hormone (ACTH) secretion. Post-mortem examination showed that the source of ACTH was small cell neuroendocrine carcinoma of the prostate, and that the patient had died from the sequelae of profound immune suppression.

KEYWORDS: Autopsy, ACTH syndrome, ectopic, clinicopathological conference, carcinoma, small cell, depression, prostatic neoplasms

\section{Case presentation}

An 84-year-old man presented to his GP with several weeks of fatigue, back pain and anorexia. Blood tests showed moderately elevated prostate-specific antigen (PSA) and creatinine. He had a history of locally invasive, but asymptomatic, prostate cancer. Ultrasound examination showed bilateral hydroureter and a prostate volume of $46 \mathrm{~mL}$. He underwent ureteric stenting, but his postoperative course was complicated by hypokalaemia, depressed mood and back pain. Magnetic resonance imaging of the back revealed no cause of his pain, and he was discharged.

Authors: ${ }^{\text {A }}$ palliative care consultant, Southern Adelaide Local

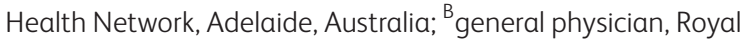
Adelaide Hospital, Adelaide, Australia; "authors contributed equally
One week later, the patient presented to the emergency department of a large public hospital with back pain radiating to his left buttock; mild bilateral proximal weakness was present, with no other neurological deficit. His depressed mood had worsened markedly and he was losing weight. He was again hypokalaemic, with associated hypertension and also hyperglycaemia, with a metabolic alkalosis. Regular psychiatric review confirmed some initial improvement in his mood following the start of treatment with mirtazapine. A computed tomography scan of pelvis showed a pelvic mass centred on the prostate with a maximal diameter of $105 \mathrm{~mm}$ and a calculated volume of $657 \mathrm{~mL}$ (Fig 1), with scattered pulmonary nodules and a heterogeneous appearance to his liver. His psychiatric and metabolic state deteriorated further. Testing found elevated serum cortisol $(7,220 \mathrm{nmol} / \mathrm{L}$ [normal range 200-700 nmol/L]), unsuppressed by low-dose dexamethasone $(10,400 \mathrm{nmol} / \mathrm{L}$ [normal $<50 \mathrm{nmol} / \mathrm{L}])$. His 24 -hour urinary free cortisol (>14,088 nmol [normal 55-250 nmol]) and plasma adrenocorticotropic hormone (ACTH) levels ( $87 \mathrm{pmol} / \mathrm{L}$ [normal 2-12 pmol/L]) were also abnormal, consistent with a diagnosis of Cushing's syndrome. His condition deteriorated rapidly and he died of sepsis, despite treatment with broad-spectrum antibiotics. He died after 3 weeks in the second hospital and only 6 weeks after admission under his private urologist.

Autopsy was performed to clarify the histology of the pelvic mass and the source of the ectopic ACTH. Post-mortem examination revealed a large mass with invasion of the pelvic sidewalls and bilateral ureters. Histologic examination revealed an invasive prostatic acinar adenocarcinoma with areas of small cell neuroendocrine carcinoma (Fig 2). There were innumerable metastatic tumour deposits within the liver, lung nodules 